\title{
Man kann Scheiße also doch aufpolieren
}

Während einer Nachtschicht in einem britischen Krankenhaus wurde ein House Officer - entsprechend etwa einem deutschen PJ-Studenten - in den Operationssaal gerufen. Dort wurde eben ein Patient wegen eines Dickdarmileus aufgrund eines Fäkaloms laparotomiert. Nach dem Eingriff erbot sich der freundliche junge Mann, noch etwas im OP aufzuräumen. Die Schwester meinte, sie würde das schon übernehmen, er solle sich aber Handschuhe anziehen und das geborgene gute Stück reinigen, damit es gut fotografiert werden könnte. Der junge Mann ging gleich zu Werke und widerlegte en passant das englische Sprichwort: „You can't polish a turd!“ Erstaunlicherweise gibt es dafür im Deutschen keine Entsprechung, obwohl wir prinzipiell dieser Substanz doch auch sehr zugetan sind. Sinngemäß ist gemeint, dass aus einer prinzipiell schlechten Sache auch mit viel Mühe nichts Gutes werden kann. Da hatten wir uns doch lieber an das Tierreich: „Wenn man einem Schwein Flügel anklebt, ist es noch kein Adler."

Prof. Dr. med. H. S. FüeßI

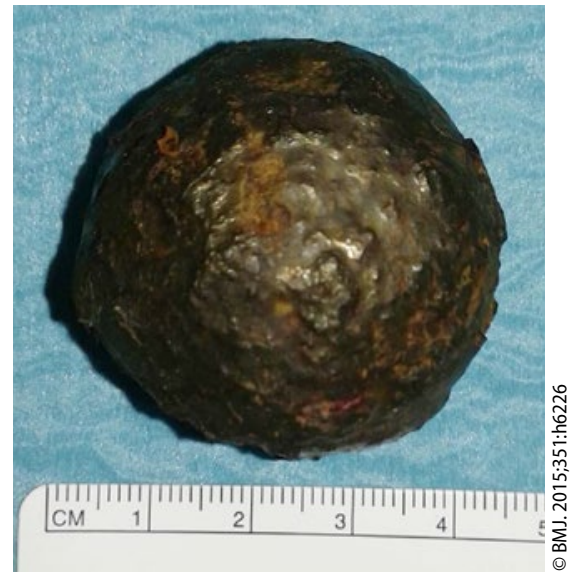

Gewissenhaft poliertes Fäkalom.

\section{Gefriert bei Furcht wirklich das Blut in den Adern?}

\author{
Eine Untersuchung an der Universität Leiden (dem Entdeckungsort der Faktor-V-Leiden- \\ Mutation!) bringt Hinweise, dass an der Redensart vom gefrorenen Blut in den Adern bei \\ Horrorerlebnissen doch ein Körnchen Wahrheit sein könnte.
}

\begin{abstract}
„O Herrin, wein' dich aus, - o weine doch - / Der Jammer greift dir tödtend an das Herz, - / Dir stockt das Blut"

- Friedrich de La Motte Fouqué, „Der Held des Nordens"(1810)
\end{abstract}

- Seit Jahrhunderten und in allen europäischen Sprachen gibt es die Vorstellung, dass bei extremen Angstsituationen das Blut in den Augen gefriert oder starr wird. Ob an dieser Vorstellung etwas wahr ist, wurde nie überprüft. Zwar gibt es Untersuchungen über verstärkte Gerinnungsaktivitäten bei Patienten mit chronischer Angststörung oder auch bei jungen Freiwilligen nach dem BungeeSpringen. Allerdings entsprechen diese Situationen nicht den Szenarien, die bei dieser Formulierung gemeint ist.

Von 24 jungen gesunden Freiwilligen sahen sich 14 zunächst einen Horrorfilm („Insidious“) und dann einen emotional nicht belastenden Fortbildungsfilm („Ein Jahr in der Champagne“) an. Zehn der Probanden besuchten die beiden Filmvorführungen in umgekehrter Reihenfolge. Die Teilnehmer wussten weder, was sie bei der Filmvorführung erwartete, noch kannten sie den Sinn der Untersuchung. Beide Filme dauerten etwa 90 Minuten und wurden im Abstand von genau einer Woche gezeigt.

Vor und nach den Vorstellungen wurde den Teilnehmern Blut abgenommen und Faktor VIII, D-Dimer, ThrombinAntithrombin-Komplexe und Antithrombin-Fragmente $1+2$ bestimmt. Das Ausmaß der von den Teilnehmern empfundenen Angst wurde anhand einer zehnteiligen visuellen Analogskala bestimmt.

Nach dem Horrorfilm wurden Scores zwischen 4,7 und 6,1 auf der Angstskala angegeben. Die Differenz zwischen dem vorherigen und dem nachherigen Faktor-VIII-Wert war beim Horrorfilm mit einem Mittelwert von 11,1 IU/dl signifikant höher als bei dem entspannten Film. Alle anderen hinweisenden Parameter auf eine erhöhte Gerinnungsneigung blieben dagegen unverändert.

Die Erhöhung des Faktor-VIII-Werts könnte klinisch relevant sein, da mit je- dem Anstieg um 10 IU/dl ein um 17\% erhöhtes Risiko für eine Thrombose zu erwarten ist. Da sich die übrigen Parameter nicht veränderten, kann man davon ausgehen, dass bei akuter Furcht zwar die Gerinnungskaskade angeregt wird, dies aber nicht zur Bildung von Thrombin und Fibrin führt.

- Nemeth B et al. Bloodcurdling movies and measures of coagulation: fear factor crossover trial. BMJ. 2015;351:h6367

\section{KOMMENTAR}

Bei einem durchschnittlichen Angstgrad von 5,4 auf einer zehnteiligen Skala scheint bei der Verängstigung doch noch ziemlich viel Luft nach oben zu bestehen. Entweder war das Publikum zu hartgesotten oder waren die Reize zu schwach. „Insidious" ist ein typischer Horrorfilm über eine Familie, in deren neuem Haus es spukt. Er erhielt in den USA die Altersfreigabe PG-13, eignet sich also auch für junge Teenager.

So ganz von der Hand zu weisen ist die Redensart vom in den Adern erstarrten Blut indes nicht.

Prof. Dr. med. H. S. FüeßI 